\title{
Surveying Patients with Smart Devices
}

\author{
Patrick Lamber \\ Free University of Bozen-Bolzano \\ patrick.lamber@lamber.info \\ Laura Napolitano \\ Free University of Bozen-Bolzano \\ laura.napolitano@stud-inf.unibz.it
}

\author{
Manfred Mitterer \\ Hospital Franz Tappeiner Meran \\ manfred.mitterer@asbmeran-o.it \\ Francesco Ricci \\ Free University of Bozen-Bolzano \\ francesco.ricci@unibz.it
}

\begin{abstract}
In this paper we investigate the impact of patient's profile on the usability of filling clinical questionnaires on smart devices. Our study was conducted in an oncological day hospital, where the EORTC QLQ-C30 quality of life questionnaire is administered to outpatients using computerized devices (smartphone, tablet, and laptop) and a paper form. We show that all the devices are evaluated as usable by the patients, under the dimension of easiness of use, and provided information. Moreover, we show that the patient's cognitive functioning $(C F)$ impacts negatively on the evaluated usability of laptop-based surveys, suggesting that $C F$ must be taken into account in the GUI design. Finally, we illustrate that the patient age and her technological skills also have a negative impact on the evaluated usability.
\end{abstract}

\section{Introduction}

Computerized questionnaires can efficiently collect patients' subjective clinical data. For instance, patients under treatment in cancer clinical trials can enter their quality of life subjective assessment using the European Organization for Research and Treatment of Cancer Quality of Life Questionnaire-C30 (EORTC QLQ-C30) [1]. Also the benefits or side-effects of their prescribed medical treatments can be monitored with other ad hoc questionnaires.

Hence, extensive research was conducted in the past to assess if computerized clinical questionnaires could successfully substitute traditional paper forms. Many studies (e.g., $[9,11])$, demonstrated the reliability of computerized questionnaires running on desktop and touchscreen com- puters with respect to their paper-based equivalents. Some researches also focused on the reliability of PDAs [3, 4], tablets [10], and web-based forms [7]. Other studies were concerned with the acceptability of computerized questionnaires. A preference for computerized versions over paperbased forms is illustrated in [3,12], while the usefulness of computerized questionnaires was obserbed in $[2,7,10]$.

Notwithstanding this considerable amount of work, no previous study has analyzed the usability dimensions of computerized questionnaires, nor the impact of the patient's clinical profile on the system usability evaluation. In this paper we cover these gaps and present the results of an explorative user study conducted in an oncological day hospital. The study examined a traditional paper form and three QLQ-C30 filling applications especially designed for laptop, touchscreen smartphone, and tablet devices. Our research goals were twofold:

1. Measure the patient evaluated ease of use, the quality of the given information, and the overall usability of the four considered tools, and check if there are significant differences among them.

2. Make explicit the influence of the patient's profile on system usability; in particular we focused on sociopsycho-physical indicators derived from the QLQ-C30 questionnaire, the patient's age, and acquaintance with technology.

Our study shows that computerized questionnaire are considered usable by the patients; in particular with respect to ease of use, and the quality of the provided information. More interestingly, we demonstrate that the patient's evaluation of the usability of the laptop-based application 


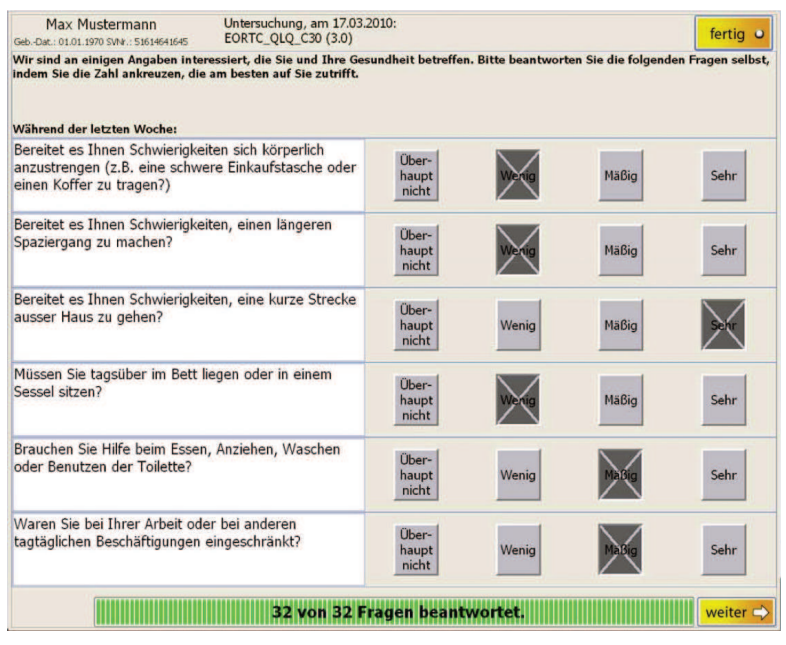

Figure 1. QLQ-C30 on the tablet.

is significantly correlated with her CF. Some other preliminary results suggest that the profile is also influential on the usability of the survey applications running on the other (smart) devices, and that the profile has a different impact depending on the device.

The rest of the paper is organized as follows. Section 2 describes the four variants (paper form, tablet, smartphone, and laptop) for the fill out of QLQ-C30 that we evaluated. Section 3 describes how the user study was organized, and discusses the obtained results. In Section 4 we formulate some conclusions, and outline future research work.

\section{Quality of life questionnaire filling}

The QLQ-C30 [1] quality of life questionnaire is a well known instrument used to assess the quality of life of cancer patients and is routinely administrated, using a paper form, in the oncological unit of the Merano hospital, i.e., where we conducted our study. We considered three alternative tools for administering the questionnaire. The first one is CHES (Computer-based Health Evaluation System) [8], a tablet-based application where the users, using a stylus instead of a pen, enter their replies by performing the same actions required by the paper-based form (see Figure 1).

The second application (see Figure 2) is web-based, and users access it through a standard web browser (on a MacBook laptop). The application adapts the graphical user interface to the client device characteristics. All the questions are presented in a single screen, and the patient can choose the right answer from drop down lists.

MobiDay [5] is the third tool: a mobile application that supports QLQ-C30 filling on a smartphone (Nokia N97 touchscreen). Figure 3 shows the screen for entering a

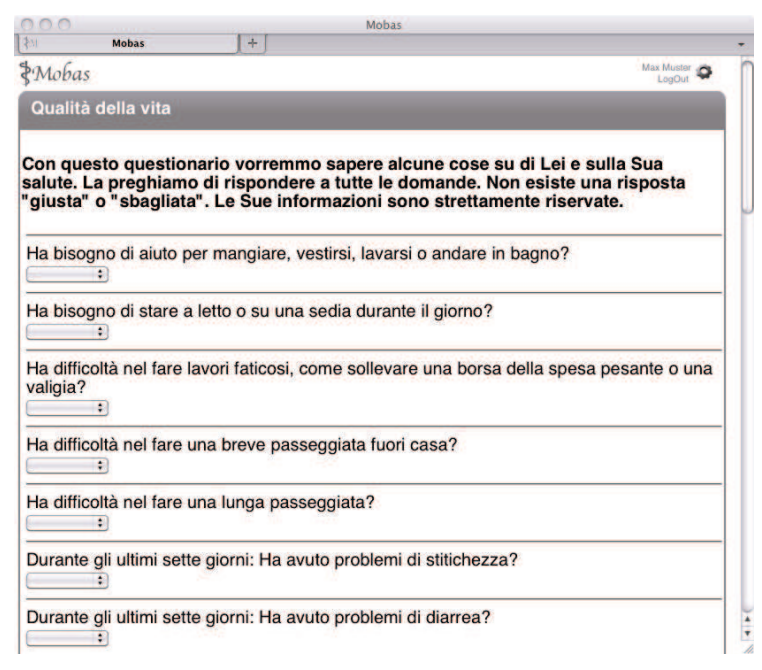

Figure 2. QLQ-C30 on the laptop.

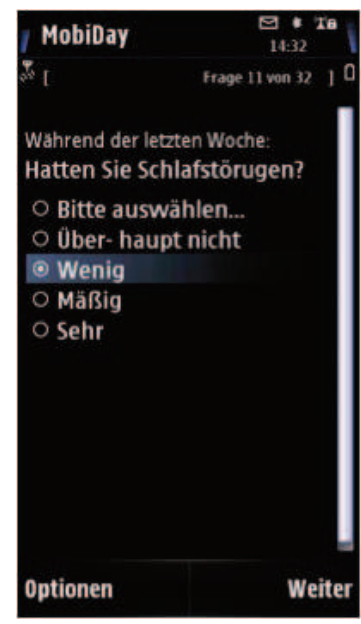

Figure 3. QLQ-C30 on the smartphone.

QLQ-C30 question. When designing the MobiDay interface we considered the limitations imposed by the small screen, but also the fact that patients access the system in an environment where disturbances and interruptions are common. For these reasons MobiDay presents the questions one by one and a patient can suspend and restart the task if necessary.

\section{User study}

The goal of the field study was to measure the usability of the questionnaire filling interfaces running on the three mentioned devices and that of the paper form, and to investigate the influence of the patient's (clinical) profile on the evaluated usability. The clinicians selected 74 users (see Ta- 


\begin{tabular}{|l|c|c|c|c|c|c|}
\hline & $30-39$ & $40-49$ & $50-59$ & $60-69$ & $70-80$ & total \\
\hline $\mathrm{P}$ & 2 & 3 & 3 & 3 & 3 & 14 \\
$\mathrm{~L}$ & 8 & 7 & 6 & 10 & 4 & 35 \\
$\mathrm{~S}$ & 3 & 4 & 2 & 3 & 3 & 15 \\
$\mathrm{~T}$ & 2 & 1 & 2 & 4 & 1 & 10 \\
\hline total & 15 & 15 & 13 & 20 & 11 & 74 \\
\hline
\end{tabular}

Table 1. Age distribution of the patients in the four groups (P: paper, L: laptop, S: smartphone, T: tablet).

\begin{tabular}{|l|l|}
\hline$Q_{1}$ & $\begin{array}{l}\text { It was simple to use the system to fill out the qual- } \\
\text { ity of life questionnaire. }\end{array}$ \\
\hline$Q_{2}$ & $\begin{array}{l}\text { The information provided by the system was ef- } \\
\text { fective in helping me to complete the filling of the } \\
\text { quality of life questionnaire. }\end{array}$ \\
\hline$Q_{3}$ & Overall, I am satisfied with the system. \\
\hline
\end{tabular}

Table 2. System usability survey.

ble 1), who were randomly assigned to one of the 4 devices.

We measured three usability dimensions (simplicity of use, quality of information, and overall satisfaction) by asking the patients to reply the post-task questions shown in Table 2. The questions were extracted from IBM CSUQ [6]. The measured average usability scores, in a range from 1 to 5 , are very high for all the questions and devices (see Figure 4). Besides, we observe that the scores reflect the systems' maturity: paper-based and laptop-based applications generally score higher than smartphone and tablet. These last two devices are someway complementary: the smartphone is easier to use, perhaps because of the touch screen, while the tablet scores better in terms of information provided. We explain this by observing that the interface of

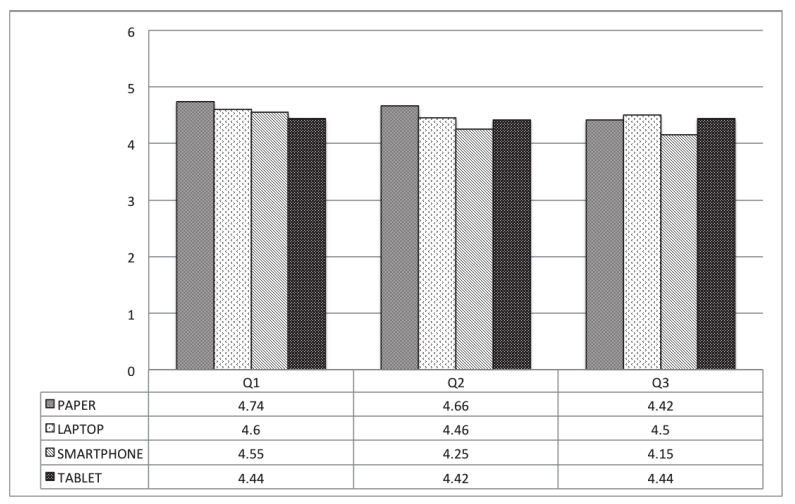

Figure 4. Usability of the four applications.

\begin{tabular}{|c|c|c|}
\hline & $C F$ & age \\
\hline$Q_{1}$ & - & -0.51 \\
\hline$Q_{2}$ & -0.41 & - \\
\hline
\end{tabular}

Table 3. Significant Spearman correlations between the patient's CF and age, and the usability variables $(\alpha=0.05)$.

MobiDay is rather basic, and the reduced size of the phone screen makes full explanations more difficult to show. We performed the non parametric Mann-Whitney test among all the pairs of systems to verify if there are statistically significant differences in the scores. We found that the smartphone is inferior to the paper form $(\alpha=0.05)$ with respect to the system informativeness. This can depend on the fact that on the smartphone the questions are presented one by one, while the general instructions for filling out the QLQ-C30 are shown only at the beginning.

We then investigated if specific (clinical) features of the patients influenced their usability evaluation. We concentrated on the laptop as this is the only group where enough samples were collected (to assure reliable results). The considered patient's features were: physical functioning, role functioning, cognitive functioning, social functioning, emotional functioning, global health status, and age. The first six features are indicators calculated from the answers to QLQ-C30. The last feature was asked directly to the subjects. To test the influence of these features on the usability assessment we measured the Spearman correlations between their values and the patient's evaluation of the system on questions $Q_{1}, Q_{2}$, and $Q_{3}$. Table 3 summarizes the cases in which we found a significant correlation using a two-tails significance test. The laptop is considered easier to use when the patient is younger (as expected). More interestingly, the $\mathrm{CF}$ of the patient (measuring her ability to concentrate and remember) is negatively correlated with the perceived informativeness. We explain this by observing that patients having lower $\mathrm{CF}$ (and being aware of that) may tend to blame themselves, instead of the device, for the perceived usability deficiencies.

The last investigation focussed on the impact of the patient's technology skills, obtained from a pre-task survey, on the evaluation of the laptop usability. Figure 5 illustrates the average scores of the laptop-based application for each usability variable, by the two classes of subjects. Technologically skilled subjects generally scored the system higher, even tough the differences are small. We verified (Mann-Whitney non parametric test) that the differences are significant ( $\alpha=0.01)$ for all the usability factors. These results show that the unfamiliarity with technology has a negative influence on the usability evaluation. On the other hand, the high scores given also by non technolog- 


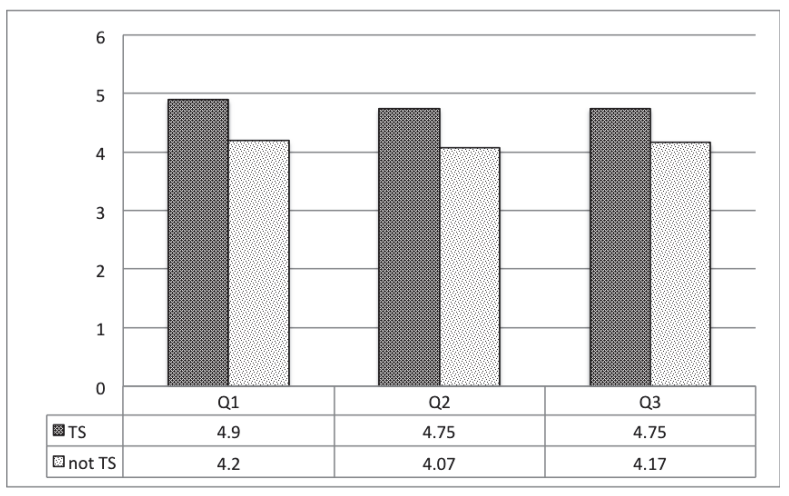

Figure 5. Average usability of the laptop wrt patient's technology skills (TS = "technologically skilled", not TS = "not technologically skilled").

ically skilled patients are illustrative of the possibility of adopting computerized questionnaires on a large scale.

We also analyzed the impact of the profile on the usability of the other devices. The paper form is considered more usable by older patients, while the global health status seems to have a negative effect on the usability of the smartphone and a positive effect on that of the paper form. However, these correlations are not statistically significant and these effects need to be further investigated on a larger sample of patients.

\section{Conclusions}

In this paper we have shown that the computerized administration of QLQ-C30 is considered usable by oncological patients, regardless the used smart device (laptop, tablet, or smartphone). In fact, all the smart devices score high and close to the paper form on all three considered usability dimensions. Only with respect to informativeness the paper-based application scored significantly better than the smartphone one (about 4\%). Moreover, we discovered that the perceived system usability is influenced by the patient's clinical profile, and we have illustrated several dependencies between user's profile and usability. On the medical side, the adoption of computerized systems was evaluated by the clinicians as extremely useful: they reported that in some cases they could took better clinical decisions based of the data collected and immediately elaborated by the smart devices.

In the future, we want to explore deeper the effect of the patient clinical profile on the system usability, focusing on smartphone and tablet. In particular, we want to better understand the implications of the found negative effects of the global health status on the perceived system usability.

\section{References}

[1] Questionnaire - quality of life. http://groups. eortc.be/qol/downloads/modules/ specimen_20qlq_c30.pdf.

[2] L. E. Carlson, M. Speca, N. Hagen, and P. Taenzer. Computerized quality-of-life screening in a cancer pain clinic. Journal of Palliative Care, 17(1):46-52, 2001.

[3] T. Heiberg, T. K. Kvien, Ø. Dale, P. Mowinckel, G. J. Aanerud, A. B. Songe-Moller, T. Uhlig, and K. B. Hagen. Daily health status registration (patient diary) in patients with rheumatoid arthritis: a comparison between personal digital assistant and paper-pencil format. Arthritis Rheum, 57(3):454-460, Apr 2007.

[4] T. K. Kvien, P. Mowinckel, T. Heiberg, K. L. Dammann, $\varnothing$. Dale, G. J. Aanerud, T. N. Alme, and T. Uhlig. Performance of health status measures with a pen based personal digital assistant. Ann Rheum Dis, 64(10):1480-1484, Oct 2005.

[5] P. Lamber, B. Ludwig, F. Ricci, F. Zini, and M. Mitterer. Message-Based Patient Guidance in Day-Hospital. In IEEE MDM 2011 - 12th IEEE International Conference on Mobile Data Management. IEEE Computer Society, 2011.

[6] J. R. Lewis. IBM computer usability satisfaction questionnaires: psychometric evaluation and instructions for use. International Journal of Human-Computer Interaction, 7:5778, January 1995.

[7] H. MacKenzie, A. Thavaneswaran, V. Chandran, and D. D. Gladman. Patient-reported outcome in psoriatic arthritis: a comparison of web-based versus paper-completed questionnaires. J Rheumatol, 38(12):2619-2624, Dec 2011.

[8] V. Meraner, J. Giesinger, G. Kemmler, S. Taucher, M. Hubalek, B. Weber, G. Rumpold, B. SpernerUnterweger, and B. Holzner. Development of a screening tool for the identification of psychooncological treatment need in breast cancer patients. Psychooncology, 18(9), 2009.

[9] S. Ramachandran, J. Lundy, and S. Coons. Testing the measurement equivalence of paper and touch-screen versions of the eq-5d visual analog scale (eq vas). Quality of Life Research, 17:1117-1120, 2008. 10.1007/s11136-008-9384-8.

[10] J. G. Richter, A. Becker, T. Koch, M. Nixdorf, R. Willers, R. Monser, B. Schacher, R. Alten, C. Specker, and M. Schneider. Self-assessments of patients via tablet pc in routine patient care: comparison with standardised paper questionnaires. Ann Rheum Dis, 67(12):1739-1741, Dec 2008.

[11] D. B. Schefte and M. L. Hetland. An open-source, selfexplanatory touch screen in routine care. validity of filling in the bath measures on ankylosing spondylitis disease activity index, function index, the health assessment questionnaire and visual analogue scales in comparison with paper versions. Rheumatology (Oxford), 49(1):99-104, Jan 2010.

[12] G. Velikova, E. P. Wright, A. B. Smith, A. Cull, A. Gould, D. Forman, T. Perren, M. Stead, J. Brown, and P. J. Selby. Automated collection of quality-of-life data: a comparison of paper and computer touch-screen questionnaires. J Clin Oncol, 17(3):998-1007, Mar 1999. 